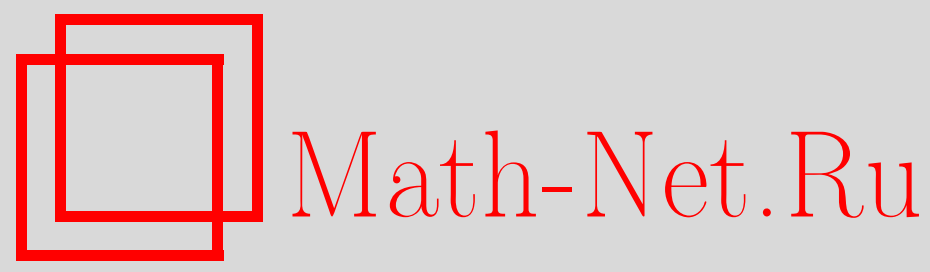

В. И. Афанасьев, Функциональная предельная теорема для остановленного случайного блуждания, достигающего высокого уровня, Дискрет. матем., 2016, том 28, выпуск 3, 313

DOI: https://doi.org/10.4213/dm1379

Использование Общероссийского математического портала Math-Net.Ru подразумевает, что вы прочитали и согласны с пользовательским соглашением http://www.mathnet.ru/rus/agreement

Параметры загрузки:

IP : 44.207 .124 .84

26 апреля 2023 г., $16: 15: 26$

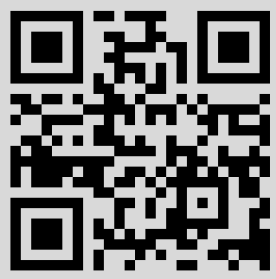




\section{Функциональная предельная теорема для остановленного случайного блуждания, достигающего высокого уровня}

() 2016 г. В.И. Афанасьев*

Для остановленного случайного блуждания с нулевым сносом, рассматриваемого при условии достижения им высокого уровня, доказана теорема о сходимости по распределению в пространстве $D[0,+\infty)$ к броуновскому прыжку в высоту.

Ключевые слова: броуновская извилина, броуновская экскурсия, броуновский прыжок в высоту, остановленное случайное блуждание, функциональные предельные теоремы

1. Пусть $X_{1}, X_{2}, \ldots$ - независимые одинаково распределенные случайные величины, удовлетворяющие условию

$$
\mathbf{E} X_{1}=0, \quad \mathbf{E} X_{1}^{2}:=\sigma^{2}, \quad 0<\sigma^{2}<+\infty .
$$

Рассмотрим случайное блуждание

$$
S_{0}=0, \quad S_{n}=\sum_{i=1}^{n} X_{i}, n \in \mathbf{N} .
$$

Пусть $\mathcal{T}$ - момент первого достижения полуоси $(-\infty, 0]$ этим блужданием, т.е.

$$
\mathcal{T}=\min \left\{n>0: S_{n} \leqslant 0\right\} .
$$

Остановленным случайным блужданием называется случайная последовательность

$$
\widetilde{S}_{n}=\left\{\begin{array}{lr}
S_{n}, & 0 \leqslant n<\mathcal{T} \\
0, & n \geqslant \mathcal{T} .
\end{array}\right.
$$

Хорошо известно, что остановленное случайное блуждание используется при описании первого периода занятости в модели массового обслуживания с единственным обслуживающим прибором (см., например, [1, том 2, гл. 6] и [2]). В задаче о разорении это блуждание описывает размер текущего капитала в игре с бесконечно богатым соперником (см., например, [1, том 1, гл. 14] и [3]).

*Место работы: Математический институт им. В. А. Стеклова Российской академии наук, e-mail: viafan@mi.ras.ru 
Остановленное случайное блуждание играет большую роль в теории ветвящихся процессов в случайной среде (ВПСС). Дело в том, что функциональные предельные теоремы для остановленного случайного блуждания и для логарифма критического ВПСС имеют схожий вид. Это позволяет находить предельные распределения для различных функционалов от критического ВПСС, используя предельные распределения для соответствующих функционалов от остановленного простого случайного блуждания. Поясним эту мысль на примере.

Пусть $W^{+}=\left\{W^{+}(t), t \geqslant 0\right\}-$ броуновская извилина, причем при $t \geqslant 1$ процесс $W^{+}(t)-W^{+}(1)$ является стандартным броуновским движением, не зависящим от процесса $\left\{W^{+}(t), t \in[0,1]\right\}$. Положим

$$
\tau=\inf \left\{t>1: W^{+}(t)=0\right\} .
$$

В [2], [5] и [6] установлено, что при $n \rightarrow \infty$

$$
\left\{\frac{\widetilde{S}_{\lfloor n t\rfloor}}{\sigma \sqrt{n}}, \frac{\widetilde{S}_{\lfloor\mathcal{T} t\rfloor}}{\sigma \sqrt{n}}, t \geqslant 0 \mid \mathcal{T}>n\right\} \stackrel{D}{\rightarrow}\left\{W_{1}^{+}(t), W_{2}^{+}(t), t \geqslant 0\right\},
$$

где $W_{1}^{+}(t)=W^{+}(t \wedge \tau), W_{2}^{+}(t)=W^{+}(\tau t \wedge \tau)$ при $t \geqslant 0$, а символ $\stackrel{D}{\rightarrow}$ означает сходимость по распределению в пространстве $D[0,+\infty) \times D[0,+\infty)$ с топологией Скорохода.

Предположим, что $\left\{\xi_{n}, n \in \mathbf{N}_{0}\right\}$ - ВПСС, задаваемый последовательностью независимых одинаково распределенных (случайных) производящих функций $\left\{f_{n}(s), n \in \mathbf{N}\right\}$. Считаем, что производящая функция $f_{n}(s), s \in[-1,1]$, определяет закон размножения частиц в $(n-1)$-м поколении, $n \in \mathbf{N}$, и $\xi_{0}=1$. Положим

$$
\varkappa_{n}=\ln f_{n}^{\prime}(1), \quad \eta_{n}=\frac{f_{n}^{\prime \prime}(1)}{\left(f_{n}^{\prime}(1)\right)^{2}}, \quad n \in \mathbf{N} \text {. }
$$

Предполагается, что процесс $\left\{\xi_{n}, n \in \mathbf{N}_{0}\right\}$ является критическим, т.е.

$$
\mathbf{E} \varkappa_{1}=0
$$

и выполнены следующие моментные ограничения:

$$
0<\mathbf{E} \varkappa_{1}^{2}:=\sigma^{2}<+\infty, \quad \mathbf{E} \ln ^{q}\left(\eta_{1} \vee 1\right)<+\infty
$$

при некотором $q>2$. Рассмотрим момент вырождения процесса $\left\{\xi_{n}\right\}$ :

$$
T=\min \left\{n: \xi_{n}=0\right\} .
$$

В [4] и [6] установлено, что при выполнении условий (2), (3) и $n \rightarrow \infty$

$$
\left\{\frac{\ln \left(\xi_{\lfloor n t\rfloor}+1\right)}{\sigma \sqrt{n}}, \frac{\ln \left(\xi_{\lfloor t T\rfloor}+1\right)}{\sigma \sqrt{n}}, t \geqslant 0 \mid T>n\right\} \stackrel{D}{\rightarrow}\left\{W_{1}^{+}(t), W_{2}^{+}(t), t \geqslant 0\right\} .
$$

Отметим, что правые части соотношений (2) и (5) совпадают. Рассмотрим некоторый функционал от критического ВПСС. Чтобы найти его предельное распределение, достаточно найти предельное распределение соответствующего функционала 
от остановленного случайного блуждания, а это можно сделать непосредственно, используя так называемое простое случайное блуждание, шаг которого $X_{1}$ принимает значения 1 и -1 с одинаковыми вероятностями, равными $1 / 2$. Подробное изложение указанного приема можно найти в [5] и [6].

В соотношениях (2) и (5) процессы рассматриваются при условии, что независимая (временна́я) переменная принимает большие значения. Перейдем к рассмотрению ситуации, когда зависимая (пространственная) переменная принимает большие значения.

Рассмотрим стандартное броуновское движение $W=\{W(t), t \geqslant 0\}$. Пусть $\tau_{x}-$ момент первого достижения состояния $x$ броуновским движением $W$ :

$$
\tau_{x}=\inf \{t>0: W(t)=x\} .
$$

Введем два момента достижения состояния 0 броуновским движением $W$. Один из них $\tau_{0}^{(1)}$ предшествует моменту $\tau_{1}$, а другой $\tau_{0}^{(2)}$ следует за этим моментом:

$$
\tau_{0}^{(1)}=\sup \left\{t \in\left[0, \tau_{1}\right]: W(t)=0\right\}, \tau_{0}^{(2)}=\inf \left\{t>\tau_{1}: W(t)=0\right\} .
$$

Броуновским прыжком в высоту называется случайный процесс $W_{0}^{\uparrow}=$ $\left\{W_{0}^{\uparrow}(t), t \geqslant 0\right\}$, определяемый формулой

$$
W_{0}^{\uparrow}(t)=W\left(\left(\tau_{0}^{(1)}+t\right) \wedge \tau_{0}^{(2)}\right), t \geqslant 0 .
$$

Разнообразные свойства этого процесса изложены в [9].

Основным результатом данной работы является следующая функциональная предельная теорема. Пусть при $x>0$

$$
\mathcal{T}_{x}=\min \left\{t: \widetilde{S}_{t}>x\right\}
$$

Теорема 1. Если выполнены условия (1), то при $n \rightarrow \infty$

$$
\left\{\frac{\widetilde{S}_{\lfloor n t\rfloor}}{\sigma \sqrt{n}}, t \geqslant 0 \mid \mathcal{T}_{\sigma \sqrt{n}}<+\infty\right\} \stackrel{D}{\rightarrow} W_{0}^{\uparrow} .
$$

Отметим, что аналогичный результат для критического ВПСС установлен в [8] и [9]. Положим при $x>0$

$$
T_{x}=\min \left\{t: \ln \xi_{t}>x\right\} .
$$

Если выполнены условия (3) и (4), то при $x \rightarrow \infty$

$$
\left\{\frac{\ln \left(\xi_{\left\lfloor t \sigma^{-2} x^{2}\right\rfloor}+1\right)}{x}, t \geqslant 0 \mid T_{x}<+\infty\right\} \stackrel{D}{\rightarrow} W_{0}^{\uparrow} .
$$

Из соотношения (6), делая замену $n=\sigma^{-2} x^{2}$, получаем, что при $n \rightarrow \infty$

$$
\left\{\frac{\ln \left(\xi_{\lfloor t n\rfloor}+1\right)}{\sigma \sqrt{n}}, t \geqslant 0 \mid T_{\sigma \sqrt{n}}<+\infty\right\} \stackrel{D}{\rightarrow} W_{0}^{\uparrow} .
$$


2. Доказательство теоремы 1. Нам потребуется несколько вспомогательных утверждений. Сначала отметим, что если выполнены условия (1), то (см., например, $[1$, том 2 , гл. 12] и [3]) при $n \rightarrow \infty$

$$
\begin{gathered}
\mathbf{P}(\mathcal{T}>n) \sim \frac{c_{1}}{\sqrt{n}}, \\
\mathbf{P}\left(\mathcal{T}_{\sigma \sqrt{n}}<+\infty\right) \sim \frac{c_{2}}{\sqrt{n}},
\end{gathered}
$$

где $c_{1}$ и $c_{2}-$ положительные постоянные, причем $c_{2} / c_{1}=\sqrt{\pi / 2}$.

Лемма 1. Если выполнены условия (1), то

$$
\lim _{\varepsilon \rightarrow 0} \limsup _{n \rightarrow \infty} \mathbf{P}\left(\mathcal{T} \leqslant \varepsilon n \mid \mathcal{T}_{\sigma \sqrt{n}}<+\infty\right)=0 .
$$

Доказательство. Заметим, что

$$
\begin{gathered}
\mathbf{P}\left(\mathcal{T} \leqslant \varepsilon n, \mathcal{T}_{\sigma \sqrt{n}}<+\infty\right)=\sum_{m=1}^{\lfloor\varepsilon n\rfloor} \mathbf{P}\left(\mathcal{T} \leqslant \varepsilon n, \mathcal{T}_{\sigma \sqrt{n}}=m\right)= \\
=\sum_{m=1}^{\lfloor\varepsilon n\rfloor} \int_{\sigma \sqrt{n}+}^{+\infty} \mathbf{P}\left(\max _{i \leqslant\lfloor\varepsilon n\rfloor-m}\left(-S_{i}\right) \geqslant y\right) d \mathbf{P}\left(\mathcal{T}>m, \mathcal{T}_{\sigma \sqrt{n}}>m-1, S_{m} \leqslant y\right) .
\end{gathered}
$$

Очевидно, что при $y \in(\sigma \sqrt{n},+\infty)$ и $1 \leqslant m \leqslant\lfloor\varepsilon n\rfloor$

$$
\mathbf{P}\left(\max _{i \leqslant\lfloor\varepsilon n\rfloor-m}\left(-S_{i}\right) \geqslant y\right) \leqslant \mathbf{P}\left(\max _{i \leqslant\lfloor\varepsilon n\rfloor}\left(-S_{i}\right) \geqslant \sigma \sqrt{n}\right) .
$$

Таким образом,

$$
\begin{gathered}
\mathbf{P}\left(\mathcal{T} \leqslant \varepsilon n, \mathcal{T}_{\sigma \sqrt{n}}<+\infty\right) \leqslant \\
\leqslant \mathbf{P}\left(\max _{i \leqslant\lfloor\varepsilon n\rfloor}\left(-S_{i}\right) \geqslant \sigma \sqrt{n}\right) \sum_{m=1}^{\lfloor\varepsilon n\rfloor} \mathbf{P}\left(\mathcal{T}>m, \mathcal{T}_{\sigma \sqrt{n}}>m-1, S_{m}>\sigma \sqrt{n}\right)= \\
=\mathbf{P}\left(\max _{i \leqslant\lfloor\varepsilon n\rfloor}\left(-S_{i}\right) \geqslant \sigma \sqrt{n}\right) \sum_{m=1}^{\lfloor\varepsilon n\rfloor} \mathbf{P}\left(\mathcal{T}>m, \mathcal{T}_{\sigma \sqrt{n}}=m\right) \leqslant \\
\leqslant \mathbf{P}\left(\max _{i \leqslant\lfloor\varepsilon n\rfloor}\left(-S_{i}\right) \geqslant \sigma \sqrt{n}\right) \mathbf{P}\left(\mathcal{T}_{\sigma \sqrt{n}}<+\infty\right)
\end{gathered}
$$

и, следовательно,

$$
\mathbf{P}\left(\mathcal{T} \leqslant \varepsilon n \mid \mathcal{T}_{\sigma \sqrt{n}}<+\infty\right) \leqslant \mathbf{P}\left(\max _{i \leqslant\lfloor\varepsilon n\rfloor}\left(-S_{i}\right) \geqslant \sigma \sqrt{n}\right) .
$$

По принципу инвариантности Донскера-Прохорова

$$
\lim _{n \rightarrow \infty} \mathbf{P}\left(\max _{i \leqslant\lfloor\varepsilon n\rfloor}\left(-S_{i}\right) \geqslant \sigma \sqrt{n}\right)=\mathbf{P}\left(\sup _{t \leqslant \varepsilon} W(t) \geqslant 1\right) .
$$


Поэтому

$$
\begin{gathered}
\lim _{\varepsilon \rightarrow 0} \lim _{n \rightarrow \infty} \mathbf{P}\left(\max _{i \leqslant\lfloor\varepsilon n\rfloor}\left(-S_{i}\right) \geqslant \sigma \sqrt{n}\right)=\lim _{\varepsilon \rightarrow 0} \mathbf{P}\left(\sup _{t \leqslant \varepsilon} W(t) \geqslant 1\right)= \\
=\lim _{\varepsilon \rightarrow 0} \mathbf{P}\left(\sup _{t \leqslant 1} W(t) \geqslant \frac{1}{\sqrt{\varepsilon}}\right)=0
\end{gathered}
$$

Из соотношений (9) и (10) следует утверждение леммы.

Пусть $W_{0}^{+}=\left\{W_{0}^{+}(t), t \geqslant 0\right\}-$ броуновская экскурсия, причем $W_{0}^{+}(t)=0$ при $t \geqslant 1$. Следующее утверждение установлено в [2], [5] и [7].

Лемма 2. Распределение случайного процесса $\left\{W_{1}^{+}(t), W_{2}^{+}(t), t \geqslant 0\right\}$ совпадает $c$ распределением процесса

$$
\left\{\frac{W_{0}^{+}\left(\alpha^{2} t\right)}{\alpha}, \frac{W_{0}^{+}(t)}{\alpha}, t \geqslant 0\right\},
$$

где $\alpha$ - случайная величина, равномерно распределенная на интервале $(0,1)$ и не зависящая от прощесса $W_{0}^{+}$.

Положим

$$
h_{0}^{+}=\sup _{t \in[0,1]} W_{0}^{+}(t)
$$

Для каждого $v \in(0,+\infty)$ введем случайный процесс $Z_{v}=\left\{Z_{v}(t), t \geqslant 0\right\}$ по формуле

$$
Z_{v}(t)=\frac{W_{0}^{+}\left(v^{2} t\right)}{v}, \quad t \geqslant 0 .
$$

Следующий результат установлен в [9].

Лемма 3. Для произвольного борелевского множества $A$ пространства $C[0,+\infty)$

$$
\mathbf{P}\left(W_{0}^{\uparrow} \in A\right)=\sqrt{\frac{2}{\pi}} \int_{0}^{+\infty} \mathbf{P}\left(Z_{v} \in A, h_{0}^{+}>v\right) d v .
$$

Приступим к непосредственному доказательству теоремы 1 . Пусть $f$ - непрерывная ограниченная числовая функция, заданная на пространстве $D[0,+\infty)$. Для каждого $n \in \mathbf{N}$ введем случайные процессы $Y_{n}$ и $\widetilde{Y}_{n}:$ при $t \geqslant 0$

$$
Y_{n}(t)=\frac{\widetilde{S}_{\lfloor n t\rfloor}}{\sigma \sqrt{n}}, \quad \widetilde{Y}_{n}(t)=\frac{\widetilde{S}_{\lfloor\mathcal{T} t\rfloor}}{\sigma \sqrt{n}} .
$$

Достаточно показать, что

$$
\lim _{n \rightarrow \infty} \mathbf{E}\left(f\left(Y_{n}\right) \mid \mathcal{T}_{\sigma \sqrt{n}}<+\infty\right)=\mathbf{E} f\left(W_{0}^{\uparrow}\right) .
$$


В силу леммы 3 это равносильно равенству

$$
\lim _{n \rightarrow \infty} \mathbf{E}\left(f\left(Y_{n}\right) \mid \mathcal{T}_{\sigma \sqrt{n}}<+\infty\right)=\sqrt{\frac{2}{\pi}} \int_{0}^{+\infty} \mathbf{E}\left(f\left(Z_{v}\right) ; h_{0}^{+}>v\right) d v .
$$

Заметим, что

$$
\mathbf{E}\left(f\left(Y_{n}\right) ; \mathcal{T}_{\sigma \sqrt{n}}<+\infty\right)=E_{1}(n, \varepsilon)+E_{2}(n, \varepsilon),
$$

где

$$
\begin{aligned}
& E_{1}(n, \varepsilon)=\mathbf{E}\left(f\left(Y_{n}\right) ; \mathcal{T} \leqslant \varepsilon n, \mathcal{T}_{\sigma \sqrt{n}}<+\infty\right), \\
& E_{2}(n, \varepsilon)=\mathbf{E}\left(f\left(Y_{n}\right) ; \mathcal{T}>\varepsilon n, \mathcal{T}_{\sigma \sqrt{n}}<+\infty\right) .
\end{aligned}
$$

Ввиду леммы 1 и ограниченности функции $f$

$$
\lim _{\varepsilon \rightarrow 0} \limsup _{n \rightarrow \infty} \frac{E_{1}(n, \varepsilon)}{\mathbf{P}\left(\mathcal{T}_{\sigma \sqrt{n}}<+\infty\right)}=0 .
$$

Изучим $E_{2}(n, \varepsilon)$. Отметим, что

$$
E_{2}(n, \varepsilon)=\mathbf{E}\left(f\left(Y_{n}\right) ; \mathcal{T}>\varepsilon n, \sup _{t \in[0,1]} \tilde{Y}_{n}(t)>1\right)
$$

и при $t \geqslant 0$

$$
Y_{n}(t)=\sqrt{\varepsilon} Y_{\varepsilon n}\left(\frac{t}{\varepsilon}\right), \quad \tilde{Y}_{n}(t)=\sqrt{\varepsilon} \tilde{Y}_{\varepsilon n}(t)
$$

Следовательно,

$$
\begin{gathered}
\frac{E_{2}(n, \varepsilon)}{\mathbf{P}(\mathcal{T}>\varepsilon n)}= \\
=\mathbf{E}\left(f\left(\left\{\sqrt{\varepsilon} Y_{n^{\prime}}\left(\frac{t}{\varepsilon}\right), t \geqslant 0\right\}\right) I_{\left\{\sup _{t \in[0,1]} \widetilde{Y}_{n^{\prime}}(t)>1 / \sqrt{\varepsilon}\right\}} \mid \mathcal{T}>n^{\prime}\right),
\end{gathered}
$$

где $I_{A}-$ индикатор события $A$ и $n^{\prime}=\varepsilon n$. Применяя к (14) соотношение (2), получаем, что

$$
\lim _{n \rightarrow \infty} \frac{E_{2}(n, \varepsilon)}{\mathbf{P}(\mathcal{T}>\varepsilon n)}=\mathbf{E}\left(f\left\{\sqrt{\varepsilon} W_{1}^{+}\left(\frac{t}{\varepsilon}\right), t \geqslant 0\right\} ; \sup _{t \in[0,1]} W_{2}^{+}(t)>\frac{1}{\sqrt{\varepsilon}}\right) .
$$

При выводе (15) учтено, что

$$
\mathbf{P}\left(\sup _{t \in[0,1]} W_{2}^{+}(t)=\frac{1}{\sqrt{\varepsilon}}\right)=0 .
$$

В свою очередь, последнее равенство объясняется леммой 2 и абсолютной непрерывностью распределения случайной величины $\sup _{t \in[0,1]} W_{0}^{+}(t)$. В силу леммы 2

$$
\mathbf{E}\left(f\left(\left\{\sqrt{\varepsilon} W_{1}^{+}\left(\frac{t}{\varepsilon}\right), t \geqslant 0\right\}\right) ; \sup _{t \in[0,1]} W_{2}^{+}(t)>\frac{1}{\sqrt{\varepsilon}}\right)=
$$




$$
\begin{aligned}
& =\mathbf{E}\left(f\left(\left\{\sqrt{\varepsilon} \frac{W_{0}^{+}\left(\alpha^{2} t / \varepsilon\right)}{\alpha}, t \geqslant 0\right\}\right) ; \frac{\sup _{t \in[0,1]} W_{0}^{+}(t)}{\alpha}>\frac{1}{\sqrt{\varepsilon}}\right)= \\
& =\int_{0}^{1} \mathbf{E}\left(f\left(\left\{\sqrt{\varepsilon} \frac{W_{0}^{+}\left(u^{2} t / \varepsilon\right)}{u}, t \geqslant 0\right\}\right), \frac{\sup _{t \in[0,1]} W_{0}^{+}(t)}{u}>\frac{1}{\sqrt{\varepsilon}}\right) d u= \\
& =\sqrt{\varepsilon} \int_{0}^{1 / \sqrt{\varepsilon}} \mathbf{E}\left(f\left(\left\{\frac{W_{0}^{+}\left(v^{2} t\right)}{v}, t \geqslant 0\right\}\right), \sup _{t \in[0,1]} W_{0}^{+}(t)>v\right) d v .
\end{aligned}
$$

Откуда, вспоминая определение $Z_{v}$ и $h_{0}^{+}$, находим, что

$$
\begin{gathered}
\mathbf{E}\left(f\left(\left\{\sqrt{\varepsilon} W_{1}^{+}\left(\frac{t}{\varepsilon}\right), t \geqslant 0\right\}\right) ; \sup _{t \in[0,1]} W_{2}^{+}(t)>\frac{1}{\sqrt{\varepsilon}}\right)= \\
=\sqrt{\varepsilon} \int_{0}^{1 / \sqrt{\varepsilon}} \mathbf{E}\left(f\left(Z_{v}\right), h_{0}^{+}>v\right) d v .
\end{gathered}
$$

Из соотношений (15), (16) получаем, что

$$
\lim _{n \rightarrow \infty} \frac{E_{2}(n, \varepsilon)}{\mathbf{P}(\mathcal{T}>\varepsilon n)}=\sqrt{\varepsilon} \int_{0}^{1 / \sqrt{\varepsilon}} \mathbf{E}\left(f\left(Z_{v}\right), h_{0}^{+}>v\right) d v .
$$

Ввиду (7) и (8)

$$
\lim _{n \rightarrow \infty} \frac{\mathbf{P}(\mathcal{T}>\varepsilon n)}{\mathbf{P}\left(\mathcal{T}_{\sigma \sqrt{n}}<+\infty\right)}=\sqrt{\frac{2}{\pi \varepsilon}}
$$

Из (17) и (18) вытекает, что

$$
\lim _{\varepsilon \rightarrow 0} \lim _{n \rightarrow \infty} \frac{E_{2}(n, \varepsilon)}{\mathbf{P}\left(\mathcal{T}_{\sigma \sqrt{n}}<+\infty\right)}=\sqrt{\frac{2}{\pi}} \int_{0}^{+\infty} \mathbf{E}\left(f\left(Z_{v}\right), h_{0}^{+}>v\right) d v .
$$

Из (12), (13) и (19) следует требуемое соотношение (11). Теорема доказана.

3. В этом разделе устанавливаются некоторые следствия теоремы 1 . Пусть $\mu_{n}$ - время пребывания остановленного случайного блуждания над уровнем $\sigma \sqrt{n}$, т.е. $\mu_{n}=\left|\left\{i \geqslant 0: \widetilde{S}_{i}>\sigma \sqrt{n}\right\}\right|$.

Теорема 2. Если выполнены условия (1), то при $n \rightarrow \infty$

$$
\left\{\frac{\mu_{n}}{n} \mid \mathcal{T}_{\sigma \sqrt{n}}<+\infty\right\} \stackrel{D}{\rightarrow} \mu
$$

где $\mu$ - положительная случайная величина, причем

$$
\mathbf{E} \exp (-s \mu)=\frac{1}{1+\sqrt{2 s}}, \quad s>0 .
$$


Пусть $\nu_{n}$ - время пребывания остановленного случайного блуждания под уровнем $\sigma \sqrt{n}$, т.е. $\nu_{n}=\left|\left\{i \geqslant 0: 0<\widetilde{S}_{i} \leqslant \sigma \sqrt{n}\right\}\right|$.

Теорема 3. Если выполнены условия (1), то при $n \rightarrow \infty$

$$
\left\{\frac{\nu_{n}}{n} \mid \mathcal{T}_{\sigma \sqrt{n}}<+\infty\right\} \stackrel{D}{\rightarrow} \nu
$$

где $\nu$ - положительная случайная величина, причем

$$
\mathbf{E} \exp (-s \nu)=\frac{2 \sqrt{s}}{\sinh (2 \sqrt{s})}, \quad s>0
$$

Нам потребуется следующий результат.

Лемма 4. Если выполнены условия (1), то

$$
\lim _{k \rightarrow \infty} \lim _{n \rightarrow \infty} \mathbf{P}\left(\mathcal{T}>k n \mid \mathcal{T}_{\sigma \sqrt{n}}<+\infty\right)=0
$$

Доказательство. Заметим, что

$$
\mathbf{P}\left(\mathcal{T}>k n \mid \mathcal{T}_{\sigma \sqrt{n}}<+\infty\right)=\frac{\mathbf{P}(\mathcal{T}>k n)}{\mathbf{P}\left(\mathcal{T}_{\sigma \sqrt{n}}<+\infty\right)} \mathbf{P}\left(\mathcal{T}_{\sigma \sqrt{n}}<+\infty \mid \mathcal{T}>k n\right)
$$

Очевидно, что

$$
\begin{gathered}
\mathbf{P}\left(\mathcal{T}_{\sigma \sqrt{n}}<+\infty \mid \mathcal{T}>k n\right)=\mathbf{P}\left(\sup _{t \in[0,1]} \tilde{Y}_{n}(t)>1 \mid \mathcal{T}>k n\right)= \\
=\mathbf{P}\left(\sup _{t \in[0,1]} \sqrt{k} \widetilde{Y}_{k n}(t)>1 \mid \mathcal{T}>k n\right)
\end{gathered}
$$

поэтому ввиду соотношения (2)

$$
\lim _{n \rightarrow \infty} \mathbf{P}\left(\mathcal{T}_{\sigma \sqrt{n}}<+\infty \mid \mathcal{T}>k n\right)=\mathbf{P}\left(\sup _{t \in[0,1]} W_{2}^{+}(t)>\frac{1}{\sqrt{k}}\right) .
$$

Далее, в силу леммы 2

$$
\begin{gathered}
\mathbf{P}\left(\sup _{t \in[0,1]} W_{2}^{+}(t)>\frac{1}{\sqrt{k}}\right)=\mathbf{P}\left(\sup _{t \in[0,1]} W_{0}^{+}(t)>\frac{\alpha}{\sqrt{k}}\right)= \\
=\int_{0}^{1} \mathbf{P}\left(\sup _{t \in[0,1]} W_{0}^{+}(t)>\frac{u}{\sqrt{k}}\right) d u=\sqrt{k} \int_{0}^{1 / \sqrt{k}} \mathbf{P}\left(\sup _{t \in[0,1]} W_{0}^{+}(t)>v\right) d v .
\end{gathered}
$$

Следовательно,

$$
\lim _{k \rightarrow \infty} \lim _{n \rightarrow \infty} \frac{1}{\sqrt{k}} \mathbf{P}\left(\mathcal{T}_{\sigma \sqrt{n}}<+\infty \mid \mathcal{T}>k n\right)=
$$




$$
=\lim _{k \rightarrow \infty} \int_{0}^{1 / \sqrt{k}} \mathbf{P}\left(\sup _{t \in[0,1]} W_{0}^{+}(t)>v\right) d v=0 .
$$

Из соотношений (7) и (8) находим, что

$$
\lim _{n \rightarrow \infty} \frac{\mathbf{P}(\mathcal{T}>k n)}{\mathbf{P}\left(\mathcal{T}_{\sigma \sqrt{n}}<+\infty\right)}=\frac{c_{1}}{c_{2} \sqrt{k}}
$$

Из соотношений (22)-(24) следует утверждение леммы.

Доказательство (теоремы 2). Положим при $k \in \mathbf{N}$

$$
\mu_{n}(k)=\left|\left\{0 \leqslant i \leqslant k n: \widetilde{S}_{i}>\sigma \sqrt{n}\right\}\right| .
$$

Ясно, что при $u>0$

$$
\mathbf{P}\left(\frac{\mu_{n}}{n}>u \mid \mathcal{T}_{\sigma \sqrt{n}}<+\infty\right)=P_{1}(n, k)+P_{2}(n, k),
$$

где

$$
\begin{aligned}
& P_{1}(n, k)=\mathbf{P}\left(\frac{\mu_{n}}{n}>u, \mathcal{T}>k n \mid \mathcal{T}_{\sigma \sqrt{n}}<+\infty\right), \\
& P_{2}(n, k)=\mathbf{P}\left(\frac{\mu_{n}}{n}>u, \mathcal{T} \leqslant k n \mid \mathcal{T}_{\sigma \sqrt{n}}<+\infty\right) .
\end{aligned}
$$

В силу леммы 4

$$
\lim _{k \rightarrow \infty} \limsup _{n \rightarrow \infty} P_{1}(n, k)=0 .
$$

Заметим, что $\mu_{n}=\mu_{n}(k)$ при $\mathcal{T} \leqslant k n$, поэтому

$$
P_{2}(n, k)=\mathbf{P}\left(\frac{\mu_{n}(k)}{n}>u, \mathcal{T} \leqslant k n \mid \mathcal{T}_{\sigma \sqrt{n}}<+\infty\right)=P_{3}(n, k)-P_{4}(n, k),
$$

где

$$
\begin{gathered}
P_{3}(n, k)=\mathbf{P}\left(\frac{\mu_{n}(k)}{n}>u \mid \mathcal{T}_{\sigma \sqrt{n}}<+\infty\right), \\
P_{4}(n, k)=\mathbf{P}\left(\frac{\mu_{n}(k)}{n}>u, \mathcal{T}>k n \mid \mathcal{T}_{\sigma \sqrt{n}}<+\infty\right) .
\end{gathered}
$$

Снова применяя лемму 4, находим, что

$$
\lim _{k \rightarrow \infty} \limsup _{n \rightarrow \infty} P_{4}(n, k)=0 .
$$

Обозначим $\lambda_{A}$ меру Лебега борелевского множества $A$ в $\mathbf{R}$. Очевидно, что

$$
\mu_{n}(k)=\left|\left\{0 \leqslant i \leqslant k n: \widetilde{S}_{i}>\sigma \sqrt{n}\right\}\right|=n \lambda_{\left\{t \in[0, k]: Y_{n}(t)>1\right\}} .
$$

Поэтому в силу теоремы 1

$$
\lim _{n \rightarrow \infty} P_{3}(n, k)=\lim _{n \rightarrow \infty} \mathbf{P}\left(\lambda_{\left\{t \in[0, k]: Y_{n}(t)>1\right\}}>u \mid \mathcal{T}_{\sigma \sqrt{n}}<+\infty\right)=
$$




$$
=\mathbf{P}\left(\lambda_{\left\{t \in[0, k]: W_{0}^{\uparrow}(t)>1\right\}}>u\right) .
$$

Откуда, учитывая, что почти наверное $W_{0}^{\uparrow}(t)=0$ при достаточно больших (в зависимости от элементарного исхода) $t$, получаем, что

$$
\lim _{k \rightarrow \infty} \lim _{n \rightarrow \infty} P_{3}(n, k)=\mathbf{P}\left(\lambda_{\left\{t \geqslant 0: W_{0}^{\uparrow}(t)>1\right\}}>u\right) .
$$

В силу соотношений (25)-(29)

$$
\lim _{n \rightarrow \infty} \mathbf{P}\left(\frac{\mu_{n}}{n}>u \mid \mathcal{T}_{\sigma \sqrt{n}}<+\infty\right)=\mathbf{P}\left(\lambda_{\left\{t \geqslant 0: W_{0}^{\uparrow}(t)>1\right\}}>u\right) .
$$

Осталось отметить, что в [9] для случайной величины $\mu:=\lambda_{\left\{t \geqslant 0: W_{0}^{\uparrow}(t)>1\right\}}$ установлено соотношение (20). Теорема 2 доказана.

Доказательство (теоремы 3). Положим при $k \in \mathbf{N}$

$$
\nu_{n}(k)=\left|\left\{0 \leqslant i \leqslant k n: 0<\widetilde{S}_{i} \leqslant \sigma \sqrt{n}\right\}\right| .
$$

Очевидно, что при $u>0$

$$
\mathbf{P}\left(\frac{\nu_{n}}{n}>u \mid \mathcal{T}_{\sigma \sqrt{n}}<+\infty\right)=Q_{1}(n, k)-Q_{2}(n, k)+Q_{3}(n, k),
$$

где

$$
\begin{gathered}
Q_{1}(n, k)=\mathbf{P}\left(\frac{\nu_{n}(k)}{n}>u \mid \mathcal{T}_{\sigma \sqrt{n}}<+\infty\right), \\
Q_{2}(n, k)=\mathbf{P}\left(\frac{\nu_{n}(k)}{n}>u, \mathcal{T}>k n \mid \mathcal{T}_{\sigma \sqrt{n}}<+\infty\right), \\
Q_{3}(n, k)=\mathbf{P}\left(\frac{\nu_{n}}{n}>u, \mathcal{T}>k n \mid \mathcal{T}_{\sigma \sqrt{n}}<+\infty\right) .
\end{gathered}
$$

По лемме 4 при $i \in\{2,3\}$

$$
\lim _{k \rightarrow \infty} \limsup _{n \rightarrow \infty} Q_{i}(n, k)=0 \text {. }
$$

Поскольку

$$
\nu_{n}(k)=\left|\left\{0 \leqslant i \leqslant k n: 0<\widetilde{S}_{i} \leqslant \sigma \sqrt{n}\right\}\right|=n \lambda_{\left\{t \in[0, k]: 0<Y_{n}(t) \leqslant 1\right\}},
$$

то по теореме 1

$$
\lim _{n \rightarrow \infty} Q_{1}(n, k)=\mathbf{P}\left(\lambda_{\left\{t \in[0, k]: 0<W_{0}^{\uparrow}(t) \leqslant 1\right\}}>u\right)
$$

и, следовательно,

$$
\lim _{k \rightarrow \infty} \lim _{n \rightarrow \infty} Q_{1}(n, k)=\mathbf{P}\left(\lambda_{\left\{t \geqslant 0: 0<W_{0}^{\uparrow}(t) \leqslant 1\right\}}>u\right) .
$$

Из соотношений (30)-(32) вытекает, что

$$
\lim _{n \rightarrow \infty} \mathbf{P}\left(\frac{\nu_{n}}{n}>u \mid \mathcal{T}_{\sigma \sqrt{n}}<+\infty\right)=\mathbf{P}\left(\lambda_{\left\{t \geqslant 0: 0<W_{0}^{\uparrow}(t) \leqslant 1\right\}}>u\right) .
$$

Осталось заметить (см. [9]), что преобразование Лапласа случайной величины $\nu:=$ $\lambda_{\left\{t \geqslant 0: 0<W_{0}^{\uparrow}(t) \leqslant 1\right\}}$ задается формулой $(21)$. Теорема 3 доказана. 


\section{Список литературы}

1. Феллер В., Введение в теорию вероятностей и ее приложения (в двух томах), М.: Мир, 1984; англ. пер.: Feller W., An Introduction to Probability Theory and Its Applications, v. 1, 3rd edition, Wiley, 1968, 528 pp.

2. Hooghiemstra G., "Conditioned limit theorems for waiting-time processes of the M/G/1 queue", J. Appl. Probab, 20:3 (1983), 675-688.

3. Shimura M., "A class of conditional limit theorems related to ruin problem", Ann. Probab., 11:1 (1983), 40-45.

4. Афанасьев В.И., "О максимуме критического ветвящегося процесса в случайной среде", Дискретн. матем., 11:2 (1999), 86-102; англ. пер.: Afanasyev V. I., "On the maximum of a critical branching process in a random environment", Discrete Math. Appl., 9:3 (1999), $267-284$.

5. Афанасьев В.И., "О моменте достижения фиксированного уровня критическим ветвящимся процессом в случайной среде”, Дискретн. матем., 11:4 (1999), 33-47; англ. пер.: Afanasyev V. I., "On the passage time of a fixed level by a critical branching process in a random environment", Discrete Math. Appl., 9:6 (1999), 627-643.

6. Афанасьев В.И., "О моменте достижения максимума критическим ветвящимся процессом в случайной среде и остановленным случайным блужданием", Дискретн. матем., 12:2 (2000), 31-50; англ. пер.: Afanasyev V. I., "On the passage time of the maximum of a critical branching process in a random environment and of a stopped random walk", Discrete Math. Appl., 10:3 (2000), 243-264.

7. Афанасьев В.И., “Закон арксинуса для ветвящихся процессов в случайной среде и процессов Гальтона-Ватсона”, Теория вероятн. и ее примен., 51:3 (2006), 449-464; англ. пер.: Afanasyev V. I., "Arcsine Law for Branching Processes in a Random Environment and Galton-Watson Processes", Theory Probab. Appl., 51:3 (2007), 401-414.

8. Афанасьев В.И., "Теория вероятн. и ее примен.”, 54:1 (2009), 3-17; англ. пер.: Afanasyev V. I., "Invariance Principle for the Critical Branching Process in a Random Environment Attaining a High Level", 54:1 (2010), 1-13.

9. Афанасьев В.И., "Броуновский прыжок в высоту", Теория вероятн. и ее примен., 55:2 (2010), 209-225; англ. пер.: Afanasyev V. I., "Brownian High Jump", Theory Probab. Appl., 55:2 (2011), 183-197. 